\title{
Using artificial intelligence to improve medical services in China
}

\author{
Ruiyang Li ${ }^{1}$, Yahan Yang ${ }^{1}$, Shaolong $\mathrm{Wu}^{2}$, Kai Huang ${ }^{3}$, Weirong Chen ${ }^{1}$, Yizhi Liu ${ }^{1}$, Haotian Lin ${ }^{1,4}$ \\ ${ }^{1}$ State Key Laboratory of Ophthalmology, Zhongshan Ophthalmic Center, Sun Yat-sen University, Guangzhou, China; ${ }^{2}$ School of Public Health, Sun \\ Yat-sen University, Guangzhou, China; ${ }^{3}$ School of Data and Computer Science, Sun Yet-sen University, Guangzhou, China; ${ }^{4}$ Center for Precision \\ Medicine, Sun Yat-sen University, Guangzhou, China \\ Contributions: (I) Conception and design: H Lin; (II) Administrative support: H Lin; (III) Provision of study materials or patients: R Li, H Lin; (IV) \\ Collection and assembly of data: R Li, Y Yang; (V) Data analysis and interpretation: R Li, Y Yang, H Lin; (VI) Manuscript writing: All authors; (VII) \\ Final approval of manuscript: All authors. \\ Correspondence to: Professor Haotian Lin, MD, PhD. Zhongshan Ophthalmic Center, Sun Yat-sen University, Xian Lie South Road 54\#, Guangzhou, \\ China. Email: haot.lin@hotmail.com.
}

\begin{abstract}
Artificial intelligence (AI) is one hotspot of research in the field of modern medical technology. Medical AI has been applied to various areas and has two main branches including virtual and physical. Recently, Chinese State Council issued a guideline on developing AI and indicated that the widespread application of AI will improve the level of precision in medical services and achieve the intelligent medical care. Medical resources, especially the high-quality resources, are deficient across the entire health service system in China. AI technologies, such that virtual AI and telemedical technology, are expected to overcome the current limitations of the distribution of medical resources and relieve the pressure associated with obtaining high-quality health care.
\end{abstract}

Keywords: Artificial intelligence (AI); medical resource; health care; China

Submitted Aug 30, 2019. Accepted for publication Nov 19, 2019.

doi: 10.21037/atm.2019.11.108

View this article at: http://dx.doi.org/10.21037/atm.2019.11.108

\section{Introduction}

Artificial intelligence (AI) is one hotspot of research in the field of modern medical technology; it has been applied to the medical industry in various areas, including medical statistics, aiding in diagnosis and therapy, robotic surgery, medical imaging and studies of human biology (1-4). Recently, on $8^{\text {th }}$ July, 2017, the Chinese State Council issued a guideline on developing $\mathrm{AI}$ that indicates that AI plays a significant role in multiple fields, including health care. At present, there is a severe deficiency in medical resources across the entire health care system in China, and highquality health services are often available only at large hospitals, which many patients have difficulty accessing (5). As AI technology continues to improve, it can imitate and replace the primary work of human doctors and may even go beyond human ability, potentially compensating for a lack of traditional medical expertise $(1,6)$. Overall, advances in medical AI are expected to reduce the difficulties faced by patients in obtaining high-quality health care and to better disseminate medical services in China.

\section{Shortage of medical resources in China}

Traditional educational programs aimed at producing highquality doctors require a long training cycle (7). In China, the " $5+3$ " model is the main training model used to educate medical students: after five years of medical undergraduate education, residents undergo three years of standardized training and acquire their medical qualifications after passing a rigorous examination, thus the minimum time required to become a doctor is eight years (8). According to data from the World Bank, China had only 1.8 doctors per 1,000 residents in 2010 (9). Physicians have suffered a high-level of workload with low salary and poor working conditions in China (10). Most physicians work more than 40 hours per week and the average numbers of outpatients 
are up to 40 in clinics per day (11). In addition, the Chinese population and its health-care expectations are growing rapidly, resulting in a serious shortage of medical resources (12). Thus, medical resources are deficient across the Chinese health service system.

Although China has undergone constant health care reforms to alleviate the pressure caused by the lack of medical resources, an uneven distribution of medical resources still exists, and high-quality resources are still concentrated in developed cities and large hospitals, such that primary hospitals lack qualified doctors $(5,13)$. Taking ophthalmology as an example, fewer than $36 \%$ of the estimated 28,000 ophthalmologists in China perform cataract surgery, and the quality among those who do operate remains problematic, especially in rural areas (14). In addition, a reasonable hierarchical diagnosis and treatment system is still absent in China. A large number of patients are unwilling to seek health services in primary hospitals and instead seek out experts in large hospitals, indicating that a shortage of high-quality resources is a main cause for the difficulties associated with obtaining hospital services. Thus, in the traditional medical service industries, both experts and patients are in short supply, creating a bottleneck that restricts social development. Although bureaucratic reforms are urgently needed as part of the solution, this type of reform is a time-consuming and costly process (15). One option for reducing disease burden and significantly improving the quality and duration of human life is the application of modern medical technology. Due to improvements in technology, human life expectancy increased from 61.7 years in 1980 to 71.8 years in 2015 (16). Therefore, AI technology in particular is expected to usher in major breakthroughs in health care (17).

\section{Two branches of medical Al}

AI has been used in various areas of medicine and has two main branches in this context: virtual and physical (18). The virtual branch is represented by machine learning, which utilizes direct experience to expand knowledge, and includes informatics approaches ranging from information management to control of health management systems (including electronic health records) to active guidance of physicians in their diagnosis and treatment decisions (19-21). For example, an AI generated through deep convolutional neural network (CNN) algorithms can effectively screen skin disease and classify skin cancer as dermatologists (22). Other examples include pathology, analysing radiographic exams, diagnosing retinal diseases, glaucoma or cardiovascular diseases (23-27). Moreover, an ophthalmic AI agent called CC-Cruiser was recently shown to have the capacity to diagnose and provide risk stratification and treatment suggestions for congenital cataracts, a rare disease (28). Indeed, this AI system has provided breakthroughs for exploring health management system for rare diseases.

The physical branch is best represented by AI robots, which have been increasingly applied in the medical industry (29). It is expected that AI robots will replace much of the primary work of human doctors. These robots are more capable than human doctors in many areas and may one day eliminate the need for humans to perform surgery (1). Based on their function, medical robots can be divided into clinical medical robots, auxiliary rehabilitation robots, hospital service robots and medical teaching robots. Clinical medical robots include surgical robots and diagnostic and therapeutic robots. The Da Vinci system is a representative surgical robot (3). Surgical robots can assist doctors in performing operations, can greatly improve surgical accuracy, and can even be used to perform long-distance telesurgery $(30,31)$. Diagnostic and therapeutic robots, such as capsule robots and nano-robots, are intelligent micro-tools that can access different regions of the human body for medical exploration and treatment (32). Rehabilitation robots can be divided into auxiliary and therapeutic robots (33). The earliest application of an auxiliary robot was that of the Handy 1, which helped users eat meals, drink water, shave, brush their hair, and complete other simple daily activities (34). Current research examining rehabilitation robots has mainly focused on rehabilitation robot arms, intelligent wheelchairs, prostheses, rehabilitation therapy robots, among others (29). Hospital service robots include telemedicine robots, article transport robots and pharmacy service robots. These robots can be utilized to deliver blood, drugs, surgical supplies, and patient care according to user-specified instructions and with high efficiency (35). Moreover, medical teaching robots are simulation robots that can be used in clinical teaching. These robots can imitate different diseases and operation models to produce clinical simulations that help to train medical staff in clinical skills and to enhance their ability to identify responses (36).

\section{The ability of Al to improve medical services in China}

Medical AI provides the advantages of high efficiency, 
surgical accuracy, flexible action and the ability to adapt to a variety of complex external environments $(37,38)$. AI can also assist human doctors by improving diagnosis and treatment efforts, thereby elevating the quality of medical services (39). To alleviate medical resource shortages, the application of medical AI in China can improve responses to medication, decrease costs and offer more efficacious interventions (15).

The virtual AI described above can be used for opensource sharing of medical records and new findings, and aggregated data can be displayed for open-access by physicians and scientists $(1,40,41)$. Taking CC-Cruiser as an example, a collaborative cloud platform for data integration and patient screening can be developed to share patient information and data from individual hospitals, reducing the need for additional resources. The creation of a collaborative network to manage rare diseases and a cloud-based AI platform to provide medical suggestions for non-specialized hospitals are expected to improve the quality of care for patients with rare diseases (28). The advantages of using an AI platform are not limited to providing care for rare ophthalmic diseases. For example, patients with other serious conditions who are living in less-developed and remote areas can receive an initial diagnosis and testing in a local primary hospital and then use an online platform to obtain second opinions from experts at top-level hospitals $(42,43)$. In this way, patients can overcome geographical obstacles to obtaining highquality care as long as they have internet access. To some extent, this access can relieve the pressure associated with obtaining high-quality health care and facilitate the distribution of the high-quality resources that are currently only available at large hospitals in China.

Telemedical technology of AI also has significant advantages for overcoming current deficiencies in access to medical resources. Telemedicine can provide health services in extremely isolated environments and has great potential for impacting many areas of health care, including consultative, diagnostic and treatment services, as well as surgery through robotic telesurgery (44). Surgeons in large hospitals are expected to exchange medical information from one site to another and provide direction via longdistance telementoring to offer health care or education to patients or health care providers, thereby improving patient care (45). Furthermore, telemedicine clinics, which can provide high-quality outpatient service from toplevel hospitals, can solve the difficulties of rural clinics where patients face long waits to see specialists (43). This increased access would lead to improvements in time- and cost-effectiveness and enable a higher level of medical care than is currently provided in Chinese primary hospitals $(46,47)$.

The above-described applications offer the chance to make rapid breakthroughs in overcoming the current limitations associated with medical supply and demand, with the aim of providing innovative medical services and increasing clinical value to patients. In this way, intelligent medical care may be achieved in China in the near future.

\section{Limitations of medical Al technologies}

It should be noted that many AI technologies are still in the exploration stage, and various limitations must still be resolved. First, the real-world clinical applications of AI are still lack of. The outstanding performance of medical AI in experimental stage with using specific dataset with high-quality cannot represent its real-world performance $(48,49)$. The quality, performance, safety, and reliability of AI systems must be guaranteed by establishing a set of standards and validated with rigorous clinical trials. Second, relevant laws, regulations, ethical guidelines and accountability should be formulated; for example, laws regulating information security and privacy must be established to ensure that patients' medical data are protected $(18,50)$. Third, the widespread use of AI may also result in changing the traditional doctor-patient relationship, which could negatively influence the health care environment. These issues require both updated guidelines and new regulations regarding AI applications. In taking these measures, smarter and more reliable medical $\mathrm{AI}$ can be developed in the future.

\section{Conclusions}

The current lack of medical resources in China, especially high-quality resources, has created an urgent need for innovative AI technologies. The most important benefits of medical AI include increased time- and costeffectiveness, an elevated level of medical care, and a greater distribution of resources (44). Medical AI should be extended to the management of a greater number diseases, and additional efforts are needed to explore the feasibility of broader clinical implementation. The development of smarter and safer AI systems is expected to bring meaningful improvements to the entire Chinese health care system. The widespread application of AI will 
improve the level of precision in medical services in China, leading to comprehensive improvement of the quality of human life.

\section{Acknowledgments}

Funding: The principal investigator of this study (HTL) is currently supported by the National Key R \& D project (2018YFC0116500), the Key Research Plan for the National Natural Science Foundation of China Cultivation Project (91546101), the National Natural Science Foundation of China (81770967), the Fundamental Research Funds for the Central Universities (16ykjc28), the Guangdong Provincial Natural Science Foundation for Distinguished Young Scholars of China (2014A030306030), and the Guangdong Province Universities and Colleges Youth Pearl River Scholar Funded Scheme (2016). These sponsors and funding organizations had no role in the design or performance of this study.

\section{Footnote}

Provenance and Peer Review: This article was commissioned by the Guest Editors (Haotian Lin and Limin Yu) for the series "Medical Artificial Intelligent Research" published in Annals of Translational Medicine. The article was sent for external peer review organized by the Guest Editors and the editorial office.

Conflicts of Interest: All authors have completed the ICMJE uniform disclosure form (available at http://dx.doi. org/10.21037/atm.2019.11.108). The series "Medical Artificial Intelligent Research" was commissioned by the editorial office without any funding or sponsorship. HL served as the unpaid Guest Editor of the series. The other authors have no other conflicts of interest to declare.

Ethical Statement: The authors are accountable for all aspects of the work in ensuring that questions related to the accuracy or integrity of any part of the work are appropriately investigated and resolved.

Open Access Statement: This is an Open Access article distributed in accordance with the Creative Commons Attribution-NonCommercial-NoDerivs 4.0 International License (CC BY-NC-ND 4.0), which permits the noncommercial replication and distribution of the article with the strict proviso that no changes or edits are made and the original work is properly cited (including links to both the formal publication through the relevant DOI and the license). See: https://creativecommons.org/licenses/by-nc-nd/4.0/.

\section{References}

1. Hamet P, Tremblay J. Artificial intelligence in medicine. Metabolism 2017;69S:S36-S40.

2. Gulshan V, Peng L, Coram M, et al. Development and Validation of a Deep Learning Algorithm for Detection of Diabetic Retinopathy in Retinal Fundus Photographs. JAMA 2016;316:2402-10.

3. Kim DH, Kim H, Kwak S, et al. The Settings, Pros and Cons of the New Surgical Robot da Vinci Xi System for Transoral Robotic Surgery (TORS): A Comparison With the Popular da Vinci Si System. Surg Laparosc Endosc Percutan Tech 2016;26:391-6.

4. Curioni-Fontecedro A. A new era of oncology through artificial intelligence. ESMO Open 2017;2:e000198.

5. Anand S, Fan VY, Zhang J, et al. China's human resources for health: quantity, quality, and distribution. Lancet 2008;372:1774-81.

6. Obermeyer Z, Emanuel EJ. Predicting the Future - Big Data, Machine Learning, and Clinical Medicine. N Engl J Med 2016;375:1216-9.

7. Zhu J, Li W, Chen L. Doctors in China: improving quality through modernisation of residency education. Lancet 2016;388:1922-9.

8. Wang C, Qi X, Chen X, et al. The establishment of China standardized residency training system. Zhonghua Yi Xue Za Zhi 2015;95:1041-3.

9. Wang YX. On the training of young doctors in China. Quant Imaging Med Surg 2015;5:182-5.

10. Tang C, Liu C, Fang P, et al. Work-Related Accumulated Fatigue among Doctors in Tertiary Hospitals: A CrossSectional Survey in Six Provinces of China. Int J Environ Res Public Health 2019. doi: 10.3390/ijerph16173049.

11. Zhang C, Liu Y. The salary of physicians in Chinese public tertiary hospitals: a national cross-sectional and follow-up study. BMC Health Serv Res 2018;18:661.

12. Zhang L, Qiu Y, Zhang N, et al. How Difficult Doctor-Patient Relationships Impair Physicians' Work Engagement: The Roles of Prosocial Motivation and Problem-Solving Pondering. Psychol Rep 2019:33294119826887. [Epub ahead of print].

13. Yip WC, Hsiao W, Meng Q, et al. Realignment of incentives for health-care providers in China. Lancet 2010;375:1120-30. 
14. Young AL, Jhanji V, Liang YB, et al. A survey of perceived training differences between ophthalmology residents in Hong Kong and China. BMC Med Educ 2015;15:158.

15. Jiang Y, Luo L, Congdon N, et al. Who will be wielding the lancet for China's patients in the future? Lancet 2016;388:1952-4.

16. Wang HD, Naghavi M, Allen C, et al. Global, regional, and national life expectancy, all-cause mortality, and causespecific mortality for 249 causes of death, 1980-2015: a systematic analysis for the Global Burden of Disease Study 2015. Lancet 2016;388:1459-544.

17. Patel VL, Shortliffe EH, Stefanelli M, et al. The coming of age of artificial intelligence in medicine. Artif Intell Med 2009;46:5-17.

18. Yang GZ, Cambias J, Cleary K, et al. Medical robotics-Regulatory, ethical, and legal considerations for increasing levels of autonomy. Science Robotics 2017;2:eaam8638.

19. Kriegeskorte N. Deep Neural Networks: A New Framework for Modeling Biological Vision and Brain Information Processing. Annu Rev Vis Sci 2015;1:417-46.

20. Shin HC, Roth HR, Gao M, et al. Deep Convolutional Neural Networks for Computer-Aided Detection: CNN Architectures, Dataset Characteristics and Transfer Learning. IEEE Trans Med Imaging 2016;35:1285-98.

21. Kermany DS, Goldbaum M, Cai W, et al. Identifying Medical Diagnoses and Treatable Diseases by Image-Based Deep Learning. Cell 2018;172:1122-31.e9.

22. Esteva A, Kuprel B, Novoa RA, et al. Dermatologist-level classification of skin cancer with deep neural networks. Nature 2017;542:115-8.

23. Poplin R, Varadarajan AV, Blumer K, et al. Prediction of cardiovascular risk factors from retinal fundus photographs via deep learning. Nature Biomedical Engineering 2018;2:158-64.

24. Li Z, He Y, Keel S, et al. Efficacy of a Deep Learning System for Detecting Glaucomatous Optic Neuropathy Based on Color Fundus Photographs. Ophthalmology 2018;125:1199-206.

25. Li Z, Keel S, Liu C, et al. An Automated Grading System for Detection of Vision-Threatening Referable Diabetic Retinopathy on the Basis of Color Fundus Photographs. Diabetes Care 2018;41:2509-16.

26. Tang A, Tam R, Cadrin-Chenevert A, et al. Canadian Association of Radiologists White Paper on Artificial Intelligence in Radiology. Can Assoc Radiol J 2018;69:120-35.
27. Campanella G, Hanna MG, Geneslaw L, et al. Clinicalgrade computational pathology using weakly supervised deep learning on whole slide images. Nat Med 2019;25:1301-9.

28. Long E, Lin H, Liu Z, et al. An artificial intelligence platform for the multihospital collaborative management of congenital cataracts. Nat Biomed Eng 2017;1:0024.

29. Najarian S, Fallahnezhad M, Afshari E. Advances in medical robotic systems with specific applications in surgery--a review. J Med Eng Technol 2011;35:19-33.

30. Smith JA, Jivraj J, Wong R, et al. 30 Years of Neurosurgical Robots: Review and Trends for Manipulators and Associated Navigational Systems. Ann Biomed Eng 2016;44:836-46.

31. Lang JE, Mannava S, Floyd AJ, et al. Robotic systems in orthopaedic surgery. J Bone Joint Surg Br 2011;93:1296-9.

32. Shang J, Noonan DP, Payne C, et al. An articulated universal joint based flexible access robot for minimally invasive surgery. IEEE Xplore 2011:1147-52.

33. Koumpouros Y. A Systematic Review on Existing Measures for the Subjective Assessment of Rehabilitation and Assistive Robot Devices. J Healthc Eng 2016;2016. doi: 10.1155/2016/1048964.

34. Topping M. An overview of the development of Handy 1. a rehabilitation robot to assist the severely disabled. J Intell Robot Syst 2002;34:253-63.

35. Ozkil AG, Fan Z, Dawids S, et al. Service Robots for Hospitals: A Case Study of Transportation Tasks in a Hospital. IEEE Xplore 2009;289-94.

36. Turini G, Moglia A, Ferrari V, et al. Patient-specific surgical simulator for the pre-operative planning of singleincision laparoscopic surgery with bimanual robots. Comput Aided Surg 2012;17:103-12.

37. Sitapati A, Kim H, Berkovich B, et al. Integrated precision medicine: the role of electronic health records in delivering personalized treatment. Wiley Interdiscip Rev Syst Biol Med 2017;9. doi: 10.1002/wsbm.1378.

38. Mirnezami R, Ahmed A. Surgery 3.0, artificial intelligence and the next-generation surgeon. 2018;105:463-5.

39. Printz C. Artificial intelligence platform for oncology could assist in treatment decisions. Cancer 2017;123:905.

40. Printz C. Genomic sequencing of treatment-resistant metastatic breast cancer reveals key alterations. Cancer 2017;123:904.

41. Castaneda C, Nalley K, Mannion C, et al. Clinical decision support systems for improving diagnostic accuracy and achieving precision medicine. J Clin 
Bioinforma 2015;5:4.

42. Ahmed MN, Toor AS, O'Neil K, et al. Cognitive Computing and the Future of Health Care Cognitive Computing and the Future of Healthcare: The Cognitive Power of IBM Watson Has the Potential to Transform Global Personalized Medicine. IEEE Pulse 2017;8:4-9.

43. Azad N, Amos S, Milne K, et al. Telemedicine in a rural memory disorder clinic-remote management of patients with dementia. Can Geriatr J 2012;15:96-100.

44. Haidegger T, Sandor J, Benyo Z. Surgery in space: the future of robotic telesurgery. Surg Endosc 2011;25:681-90.

45. Rosser JC Jr, Young SM, Klonsky J. Telementoring: an application whose time has come. Surg Endosc 2007;21:1458-63.

Cite this article as: $\mathrm{Li} \mathrm{R}$, Yang $\mathrm{Y}$, Wu S, Huang $\mathrm{K}$, Chen $\mathrm{W}$, Liu Y, Lin H. Using artificial intelligence to improve medical services in China. Ann Transl Med 2020;8(11):711. doi: 10.21037/atm.2019.11.108
46. Hashmi S. 'Coming of Age' of artificial intelligence: evolution of survivorship care through information technology. Bone Marrow Transplant 2016;51:41-2.

47. Buvik A, Bergmo TS, Bugge E, et al. Cost-Effectiveness of Telemedicine in Remote Orthopedic Consultations: Randomized Controlled Trial. J Med Internet Res 2019;21:e11330.

48. He J, Baxter SL, Xu J, et al. The practical implementation of artificial intelligence technologies in medicine. Nat Med 2019;25:30-6.

49. Dreyer KJ, Geis JR. When Machines Think: Radiology's Next Frontier. Radiology 2017;285:713-8.

50. Char DS, Shah NH, Magnus D. Implementing Machine Learning in Health Care - Addressing Ethical Challenges. N Engl J Med 2018;378:981-3. 